\title{
Measurement and control of variable geometry during ring rolling
}

\author{
Matthew R. Arthington ${ }^{1}$, Christopher Cleaver ${ }^{2}{\text { Julian } \text { Allwood }^{3} \text { and Stephen Duncan }}^{1}$
}

\begin{abstract}
Ring-rolling is an industrial forming process for producing high-strength seamless metal rings up to $6 \mathrm{~m}$ diameter. Thick-walled cylindrical rings of material, typically metallic alloys, are compressed between two or more internal and external rollers and rotated until a target geometry, often outer diameter, is achieved. A common plant configuration is that of a pair of radially acting rollers and a pair of axial rollers, the radial-axial ring rolling (RARR) machine. The most commonly produced product geometries have an axisymmetric cross-section profile. However, during the forming process the cross section is changed significantly as it passes through each pair of rollers. This irregular shape hinders geometry state measurement and this complicates modelling and control of the process. Recent developments in sensing capabilities offer high resolution measurement of ring geometry during forming. In this work, we present advances in these sensing techniques, a numerical method for storing and predicting the ring's geometrical state and control laws to achieve a nonaxisymmetric cross-section profile in rolled rings using existing RARR plant hardware.
\end{abstract}

\section{INTRODUCTION}

Ring-rolling is an industrial metal forming process for producing high-strength rings up to $6 \mathrm{~m}$ in diameter. Industry data puts 2011 worldwide hot ring rolling production at 1.7 million tonnes, some $6 \%$ of the total forging market [1]. Typical applications include components for aerospace engines, energy turbines (including Industrial Gas Turbines and Steam Turbines), pipe fittings for oil and gas and petrochemical industrials and various engineering components in shipping, mining, construction and other industries [1]. In addition to hot ring rolling, cold ring rolling is used to produce smaller ring products such as automotive bearings. As a forging process, it produces a higher strength ring than alternative casting techniques, whilst it can be quicker and less materially wasteful than alternative forging methods [2]; ring-rolling is usually significantly faster than open-die or closed-die forging and this is the key reason it is used widely in industry.

In the radial-axial ring rolling (RARR) process, a thick walled cylindrical ring is transformed into a thinner walled and/or shorter height, larger diameter ring [3], [4], [5] by compressing the wall between rotating rollers, see Figures 3 and 4 . The actuators are the motors driving the rotation of the axial rollers and the forming roller, and the linear motion of the mandrel and the forming roller.

\footnotetext{
${ }^{1,4}$ Department of Engineering Science, University of Oxford, Parks Road, Oxford, OX1 3PJ, UK, (matthew.arthington, stephen.duncan) deng.ox.ac.uk

2,3 Department of Engineering, University of Cambridge, Trumpington Street, Cambridge, CB2 1PZ, UK, (c jc82, jma42) @cam. ac.uk
}

Manual feedback has been used for many years to control the 'roll-gap', the minimum distance between pairs of rollers, as has open-loop control. Recently, feedback control has been employed to automatically achieve the target ring diameter and thickness. In order for feedback control to work efficiently in ring rolling, sensors are required to measure geometry with sufficient resolution, accuracy and frequency. Recent work [6] has shown that digital cameras can provide real-time high-resolution geometry data of the ring in a plant frame of reference. This sensing capability has been updated in this work to include tracking of the ring rotation angle, and hence measure ring geometry with respect to material positions. This system monitors the irregular shape of the ring and permits the control of its geometry during forming.

To complement the measurement of the deforming body of the ring, a model is required to store the state of geometry and predict the movement of ring material under the applied loading of the rollers. Much work has been undertaken to provide accurate and detailed simulations of ring rolling processes. Analytical models, such as those given in [7], have been superseded in terms of detail and accuracy by numerical modelling techniques - invariably finite element analyses such as those demonstrated in numerous studies, [8], [9], [10]. Several control strategies for the RARR machine have been developed [11], [12], [13], some of which are based on feasible forming conditions, such as [14] and the reduction of defects in the deformed ring [15], [16], [17]. Many studies have focused on maintaining the stable production of the continuous cross section ring, as this is the most important industrial application of RARR.

This paper outlines a strategy for measuring and controlling the profile shape of a rolled ring in a RARR machine online. The strategy allows an adaptive control algorithm to correct shape defects and approach the target shape more closely in the presence of large model uncertainty.

\section{MeAsurement OF Ring SHAPE}

In recent work [6] the authors showed how photogrammetry can be used to obtain measurements of the boundary coordinates - both the inner and outer edges of the top surface - of a ring undergoing rolling in a RARR machine. An estimate of the ring's centre position was fed back to the controller and the speed of the axial rollers was controlled to stabilise the ring's centre position. The boundary coordinates of the ring can be used to estimate the ring's irregular shape, size and centroid position, but they don't provide these in a ring-centric frame of reference. 
In order to find the ring's shape in a ring-centric frame of reference, one must measure the boundary of the ring relative to material points in its deforming body. The ring's central axis (see Figure 1) is not expected to change direction, so two material points are required to obtain a measure of the ring's position and orientation. To do this, it is proposed that markers are applied to the ring's surface and tracked to estimate the current rotation angle of the ring.

Complexity arises in the changing shape of the ring's upper surface. Ideally this forms a circular annulus, but in practice bending forces create a non-circular shape. In addition to this, the step change in wall thickness introduced by either pair of rollers makes the ring's upper surface quite irregular.

\section{A. Ring shape features}

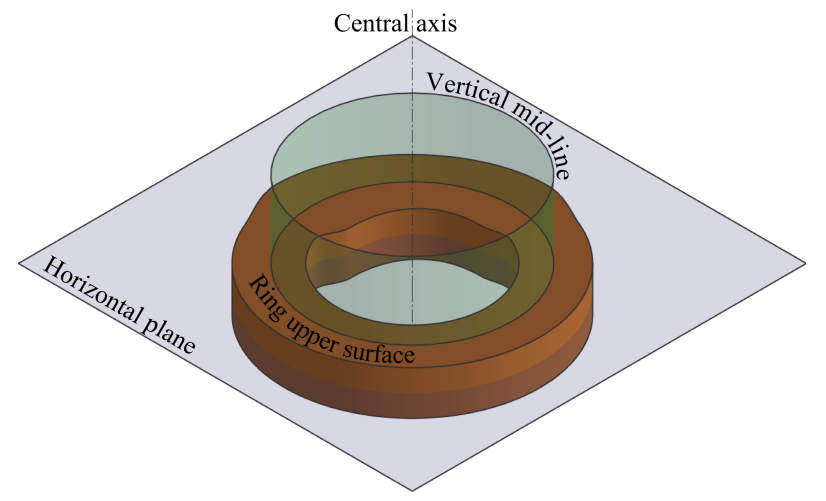

Fig. 1. Ring rolling geometry definitions.

To aid the description of the ring geometry here, two surfaces are defined, as shown in Figure 1. Firstly, the 'vertical mid-line' is the zero-wall-thickness prism (nominally cylindrical) parallel to the central axis of the ring, which divides the ring wall so that the radial distances to the inner and outer edges are equal. Secondly, the 'horizontal plane' is the plane perpendicular to the axis of the ring that divides the volume of the ring into two halves. The 'mean perimeter' is the intersection of these two surfaces. 'Mid-line' is used to describe the intersection of the upper surface of the ring and the vertical mid-line surface.

The two points used to estimate the ring's position and orientation are the ring's centre and a single point of material in the ring. To find a robust estimate of the centre of the ring, on which the central axis lies, ellipses are fitted to the inner and outer edges using a least-squares procedure, and the mean of their centres is used as the estimate of the ring's centre, $\mathbf{x}_{0}=\left[x_{c}, y_{c}\right]_{0}$. As the ring becomes distorted, a more accurate estimate of $\mathbf{x}_{0}$ is calculated by finding points on the mid-line, equidistant from the inner and outer edges along radii emanating from the first estimate of $\mathbf{x}_{0}$. The corrected $\mathbf{x}_{0}$ is then found at the centre of an ellipse fitted to the points on the mid-line.

This procedure for estimate the ring's centre provides a good estimate because the pairs of rollers have an approximately symmetric effect on the inner and outer edges

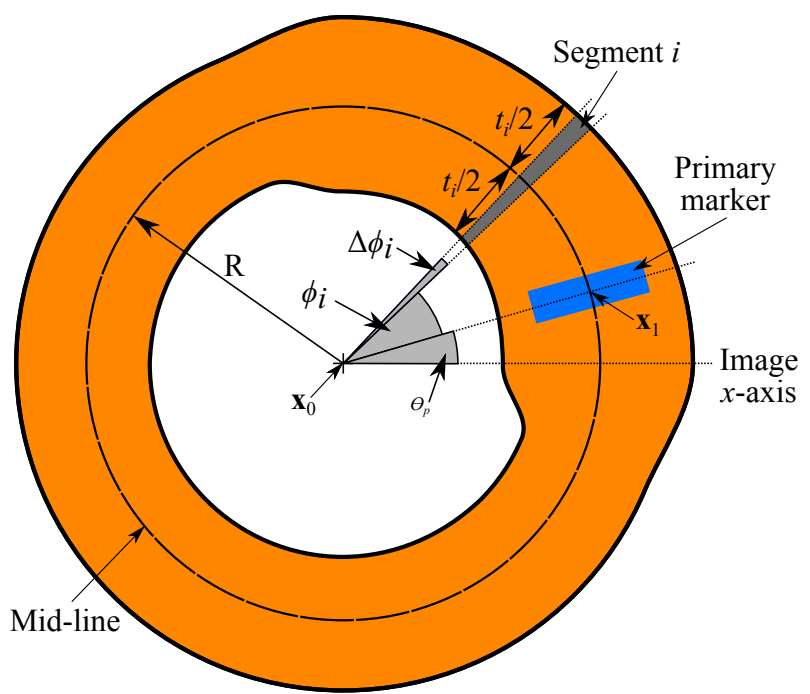

Fig. 2. Ring rolling geometry nomenclature. For clarity only the primary marker is shown.

about the mid-line, so deviations from an elliptical shape in the inner and outer edges due to wall thickness variations are eliminated. The mid-line does, however, deviate from elliptical in the presence of large bending deflections, but the process should be controlled to avoid these.

To find the position of a second point, $\mathbf{x}_{1}$, in the ring which can be tracked with respect to its deforming body, a marker, the 'primary marker', is applied to the ring's surface, as shown in Figure 2. Initially this takes the form of a line drawn radially from $\mathbf{x}_{0}$, symmetrically about the 'marker radius', between $1 / 4$ and $3 / 4$ of the distance between the inner and outer edge of the ring and having width approximately $1 / 3$ that of its length, as shown in Figure 3. In desktop-scale experiments on plasticine, this marker was applied using permanent ink, but in industry it is envisaged that hightemperature paints may be employed to the same end.

The point $\mathbf{x}_{1}$ lies on the intersection between the mid-line and the marker radius. The additional image processing steps required to find this location are:

1) Interpolate image along mid-line to obtain one dimensional intensity data.

2) Detect dip in intensity where marker lies.

3) Find tangential location of marker centre at the centroid of this dip in intensity.

The size and shape of the marker was chosen so that it would remain visible and 'trackable' during the deformation process. Initially-radial lines are distorted during ring rolling by shear strains which can slowly change their shape and/or cause their slow migration along the surface in a radial direction. It has been assumed that the intersection of the mid-line and the marker will remain close enough to a single material point in the ring for the purposes of controlling the ring's shape during rolling.

The ring body is estimated to have its centre at $\mathbf{x}_{0}$ and an orientation $\theta_{p}$ at the angle the line connecting $\mathbf{x}_{0}$ and $\mathbf{x}_{1}$ makes with the image's $\mathrm{x}$-axis, which is aligned with 
the radial roll gap direction. This position and orientation information identifies the location of the ring with respect to the rollers, and permits the control of local features in the geometry of the ring.

\section{B. Additional marker tracking}

When the primary marker is occluded by the upper axial roller, the ring's orientation is unknown. To solve this problem, additional markers are applied to the ring surface. Here, the use of 12 markers in total will be shown, but this could be changed according to resolution and frequency requirements. The primary and secondary markers are drawn in contrasting colours to distinguish them. The initially equally sized, evenly spaced, markers are shown in Figure 3 in one idealised marker arrangement.

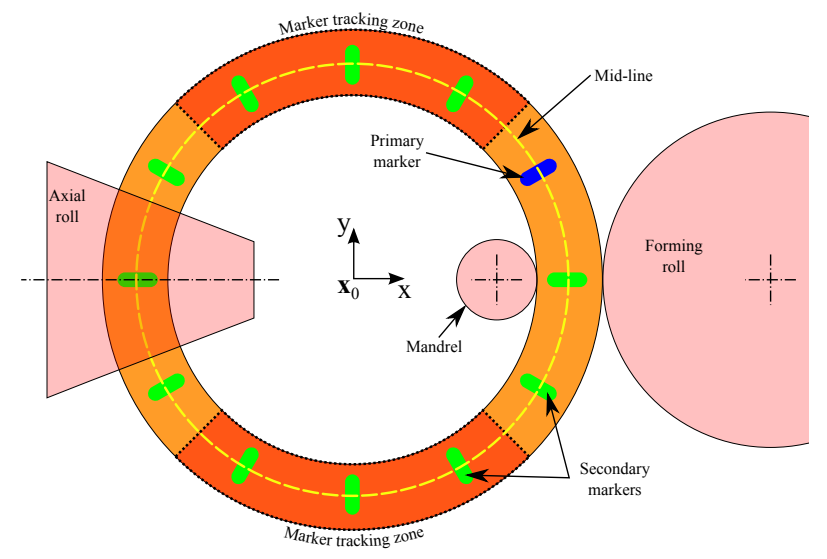

Fig. 3. Schematic plan diagram of ring with rollers and markers.

As the ring is compressed in a roll gap, the tangential distance (the distance along the mid-line) between the markers either side of that roll gap increases because of the tangential elongation, which causes the tangential velocity of material leaving the roll gap to be higher than that entering the gap. However, the tangential distances between markers inside one of the zones indicated in Figure 3 do not increase. An accurate measure of the mean ring orientation is found from the mean orientations of the markers in the upper and lower zones, $\theta_{u}$ and $\theta_{l}$ respectively, relative to $\mathbf{x}_{0}$. The mean orientation, $\theta_{s}=\left(\theta_{u}+\theta_{l}\right) / 2$, is used as the mean orientation of the whole ring while the primary marker is occluded.

$\theta_{p}$ and $\theta_{s}$ differ because $\theta_{s}$ is an average orientation, whereas the rate of increase of $\theta_{p}$ increases sharply as the primary marker moves through a roll gap. The error in ring orientation estimation while the primary marker is occluded is dependent on the size of the deformation applied by the axial rolls, but when the primary marker comes back into view it can be corrected. $\theta$ is defined as $\theta=\theta_{p}$ unless $\theta_{p}$ is unavailable, in which case $\theta=\theta_{s}$.

The purpose of tracking ring orientation is to know where each section of the ring is in relation to the rolls. In controlling radial wall thickness, one must know the input thickness of a section entering the radial roller pair so that the correct roll gap can be chosen to move the thickness value towards the target for that section. The approach taken here has been to calculate the progress around the ring by estimating the fraction of the full ring volume between the primary marker and the current roll gap. The original positions of the secondary markers on the initially-uniform ring divide the ring into 12 sections of known volumes. $\theta$ localises the roll gap position approximately, and then the volume between the roll gap and the next closest marker is estimated by integrating the product of thickness and height along the mid-line to more precisely calculate the roll gap position in the ring.

\section{STORING RING SHAPE}

In this work, simplifying assumptions are made of the real RARR process to describe the irregular geometry of a deforming ring and predict the changes to that geometry applied by radial and axial rolling. It is assumed that the ring is incompressible, and can be described sufficiently well as a set of segments with fixed volumes, each of which is a small arc in plan view, and has a rectangular cross-section, as shown in Figures 2 and 4. Using this, the geometry of irregular shapes may be modelled guaranteeing that the total volume is conserved. This discretisation is used to ensure that the controller tracks precisely which bit of the ring is about to enter either the radial or axial roll bites.

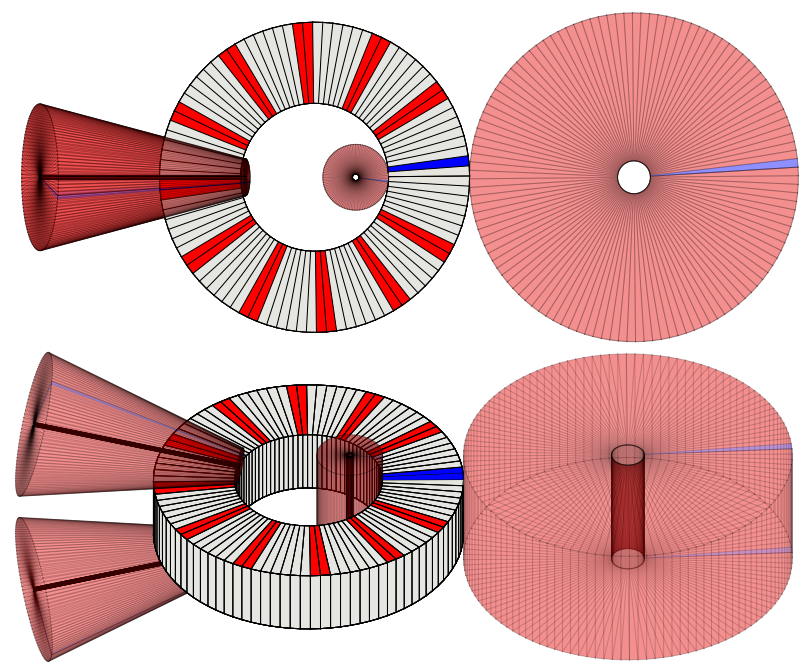

Fig. 4. The discretisation of the ring shape into radial segments. The plan and oblique views show the conical axial rollers, the internal mandrel and the external forming roller around the discretised ring. Nominal marker positions are indicated by red and blue stripes, but are not representative of the true marker shapes.

\section{A. Discretisation}

The discretisation of the ring geometry proceeds according to the following specification (using geometry parameters defined in Section II and shown in Figure 2):

1) The ring is approximated as $n$ radial segments, rectangular in cross section, at angles $\phi_{i}$ measured anticlockwise from $\theta$ about $\mathbf{x}_{0}$. Each segment has a small arc angle of $\Delta \phi_{i}=\phi_{i+1}-\phi_{i}$.

2) The centre of each segment lies on the mean perimeter. 
3) Each segment is defined by a height in the axial direction, $h_{i}$, radial thickness in the horizontal plane, $t_{i}$, and volume $v_{i}$, where $i=1 \ldots n$. The tangential length, $l_{i}$, along the mean perimeter for each node is then fixed at $l_{i}=v_{i} /\left(t_{i} h_{i}\right)$.

4) The total length of the mean perimeter is $L=$ $\sum_{i=1}^{n} l_{i}=\sum_{i=1}^{n} v_{i} /\left(t_{i} h_{i}\right)$

The graphical representation of this discretisation with a circular mean perimeter is shown in Figure 4. Initially, all $t_{i}$ are assumed equal, as are all $h_{i}$, but the $v_{i}$ need not be equal. If the rate of change of either thickness - radial or axial - with $\phi$ is large, then a higher density of segments, i.e. smaller $v_{i}$, may be used to improve the accuracy of shape approximation in that region.

\section{B. Deformation in the roll gap}

The cross section of the ring is assumed to remain rectangular in both roll gaps, each narrow segment is assumed to behave as if it were a cuboid. This assumption is not valid for a single pair of rollers, because of the fishtail phenomenon, whereby the corners of the cross section are deformed more than the centre (see, for instance, [15]), as shown in Figure 5. However, in the RARR process, each pass between one pair of rollers is followed by a pass in the orthogonal pair. This repeated flattening of the radial then horizontal sides tends to return the cross section of the ring back to a rectangular shape. Therefore, for the purposes of controlling wall thickness and height, it will be assumed that the cross section is rectangular with dimensions taken to be the average thickness and height, as the ring will tend to these over time.

In the radial roll gap it is assumed that the compressive strain causes a reduction in length in the radial direction and extrudes material in both orthogonal directions (i.e. the tangential and vertical directions) with a ratio governed by a simple model based on the current aspect ratio of the ring cross section. Similarly, in the axial roll gap any compressive strain causes reduction in length in the axial direction and expansion in the tangential and radial directions.

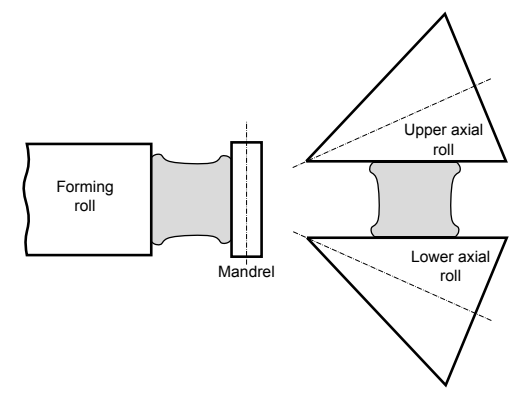

Fig. 5. The distortion of the cross section during rolling by the radial rollers (left) and axial rollers (right)

Due to the incompressibility of the material, all three orthogonal components of plastic strain must sum to zero, $\varepsilon_{v}=\varepsilon_{\text {radial }}+\varepsilon_{\text {axial }}+\varepsilon_{\text {tangential }}=0$. The extent to which the compressive strain causes extrusion in the orthogonal directions depends on the friction conditions between the rollers and the ring, giving rise to the aspect-ratio dependency. Taking the radial roll gap as an example, the compressive radial strain will cause tangential and axial elongation, unless the friction caused by the rollers is sufficiently large to constrain the axial elongation entirely, in which case all of the elongation would be tangential; a plane-strain condition, then $\varepsilon_{\text {tangential }}=-\varepsilon_{\text {radial }}$ and $\varepsilon_{\text {axial }}=0$. If there were no friction in the radial roll gap then the plastic forming state would be closer to that of uniaxial compression (plane-stress) where, for isotropic materials the plastic strain $\varepsilon_{\text {tangential }}=\varepsilon_{\text {axial }}=-\varepsilon_{\text {radial }} / 2$. The actual plastic strain ratio, $\gamma=\varepsilon_{\text {axial }} / \varepsilon_{\text {tangential }}$, is limited by $0 \leq \gamma<1$. The value of $\gamma$ for a particular material and deformation state could either be predicted offline or learned online by measuring the change in area between two markers for a given amount of strain, and hence the relative amounts of axial and tangential strain.

The controller must estimate the roll gap required to bring the state of the thickness towards the target state by the completion of the process. The rollers have finite radius, which limits the curvature in the target thickness profile. A more challenging effect of the large roller radii is experienced during forming, when the curvature of the thickness may be larger than the targeted curvature due to disturbances. To quickly compute the minimum roll gap permissible at a location without over forming any segment the set of segments, $I_{c}$, potentially in contact with the mandrel is calculated from the 'shadow' cast by the mandrel on the ring's smallest inner radius, as shown in Figure 6. All segments in the roll gap in contact with the rolls are compressed by the displacing faces of the rolls. The amount of strain developed in each segment is then used to calculate its expansion in the orthogonal directions according to the plastic flow rule in use. These estimates are used to observe the height as a function of tangential length between markers, and hence the positions of segments according to their volume.

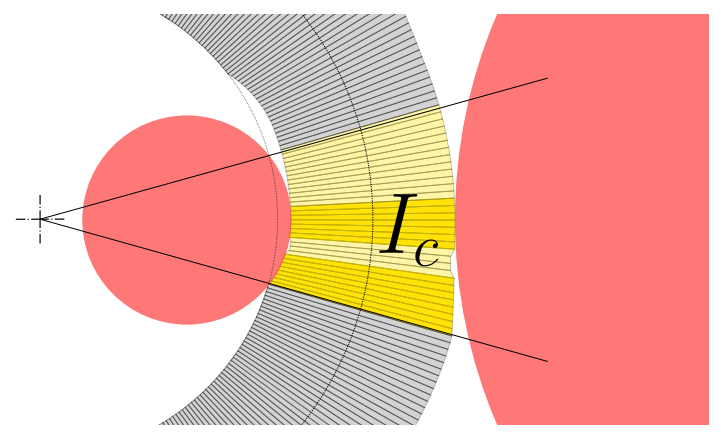

Fig. 6. The set of segments, $I_{c}$, potentially in contact with the mandrel is calculated from the 'shadow' cast by the mandrel on the ring's smallest inner radius. Within this set only some segments would be compressed by the mandrel, as shown in the darker colour.

\section{Control Strategy}

The aim of this work is to create a controller and sensing technology capable of controlling axial and radial wall thicknesses in the RARR process. The intention is for existing 
RARR hardware to be complemented by the controller. A convenient general description of the degrees of freedom in such a machine are:

1) Forming roll angular velocity, $\omega_{f}$.

2) Axial roll angular velocity (both identical), $\omega_{a}$

3) Radial roll gap distance, $g_{r}$.

4) Radial roll gap speed, $\left(\dot{g}_{r}\right)$.

5) Axial roll gap distance, $g_{a}$.

6) Axial roll gap speed, $\left(\dot{g}_{a}\right)$.

7) Forming roll position.

8) Forming roll speed.

It is assumed here that all the actuators can move to their set-points within one iteration and that the forming is performed at a speed such that the actuator limits are never exceeded.

Conventional running of a RARR machine maintains a near constant $\omega_{f}$, or at least $\omega_{f}>0$, because changing direction would increase the process duration and should not be necessary for rolling axisymmetric rings. For reasons of forming speed, the control strategy chosen here also maintains a positive $\omega_{f}$, but more complex target shapes would be accessible by permitting a reversible $\omega_{f}$.

\section{A. Target shape definition}

In this paper only circular mean perimeter shapes are considered, but it is expected that alternative convex shapes can be produced. The mean perimeter can henceforth be known as the mean circle. The target shape for a ring is specified according to the radius of the mean circle about which the axial thickness and radial thickness are specified as functions of angle about the centre of the mean circle of radius $R_{t}$. It is assumed that target shapes are feasible for production by rolling, in that they do not deviate too far from uniform rings.

The continuously specified target shape is discretised by linear interpolation into $n$ segments, which may or may not have equal volume. Each segment then has a target height, $h_{i_{t}}$, radial thickness, $t_{i_{t}}$, angular length, $\Delta \phi_{i_{t}}$, from which volume, $v_{i}$ is calculated as $v_{i}=h_{i_{t}} t_{i_{t}} \Delta \phi_{i_{t}} R_{t}$. These $n$ segments are used as the $n$ segments in the discretised model during rolling.

The total volume, used to specify the initial ring, can be found by $V=\sum v_{i}$. In addition to total volume, a suitable choice of shape for the initially-axisymmetric ring may be made by consideration of machine limitations and a minimum plastic strain limit for some segments; if the initial ring is too close to the final shape then the strain achieved may be insufficient for achieving certain microstructural properties. Initial height, radial thickness and radius, $H$, $T$ and $R_{i}$, are assumed to be larger than their respective minima.

\section{B. Segment compression scheduling}

The strategy developed here treats each volume segment of the ring as connected, but having independent shape, as in the model. As the ring rotates, the position of the next segment (the $i+1$ th segment) to reach the roll gap is known from the camera sensor, relative to the most recent surface marker (by the volume that has passed through the roll gap), and this allows its target size and shape to be determined from the target shape definition.

The amount of deformation to be applied by a roll gap to a segment is calculated based on its current size, its target size and the maximum amount of compression possible up to sticking, plus actuator limits. Sticking is where the tangential component of the friction force between the rollers and the ring is insufficient to overcome the tangential component of the compression force and the ring stops rotating while the rollerss continue to slip against its surface.

In an independent segment, the shape is transformed from its initial $H, T$ and $\Delta \phi_{i_{i}} R_{o}$ to the final $h_{i_{t}}, t_{i_{t}}$ and $\Delta \phi_{i_{t}} R_{t}$ after several passes through the roll gaps - assuming that the segment is a cuboid, the axial roll stage decreases the height and increases the radial thickness and tangential length, and the radial roll stage reduces the radial thickness while increasing the height and tangential length.

The roll gap, $g$, for the next segment has an upper bound determined by the sizes of the segments in $I_{c}$ (so that contact is not lost) and a lower bound determuned by the final target size in the gap direction for the segments in $I_{c}$, less any elastic displacement required to obtain a frictional force. Another lower bound is determined by the sticking criteria, if an estimate is available.

Within these bounds, the choice of $g$ is determined by PID control of the rate of compression (change in length towards target), or plastic strain rate. This rate is the rate per revolution, $p$, rather than w.r.t time. The number of rotations to achieve target shape is a secondary targeted value. For example, the radial roll gap, $g_{r}$, for the next segment would be determined according to Algorithm 1:

$$
\begin{aligned}
& g_{r_{\min }}=\min \left(t_{I_{c t}}\right) \\
& g_{r_{\max }}=\max \left(t_{I_{c}}\right) \\
& e_{p}=t_{i_{t}}-t_{i_{p}} \\
& g_{r}=t_{i_{p}}+K_{P} e_{p}+K_{I} \sum_{j=1}^{p} e_{j}+K_{D}\left(e_{p}-e_{p-1}\right) \\
& g_{r}=\min \left(g_{r}, g_{r_{\max }}\right) \\
& g_{r}=\max \left(g_{r}, g_{r_{\text {min }}}\right)
\end{aligned}
$$

Algorithm 1: The radial roll gap setting for the incoming segment. At the axial roller side $t$ would be interchanged for $h$. Lower case $p$ subscripts indicates the value for current pass, $t$ subscripts indicate target and $i$ subscripts denote the segment.

\section{Ring angular velocity}

The angular velocity of the ring, $\dot{\theta}$, is controlled to reach the final shape as quickly as possible without exceeding actuator limits. In traditional RARR machines, guide rolls are used to centre the ring vertically (in image coordinates), defined as $y_{c}$. Differential speed control of $\omega_{a}$ has been shown to achieve the same result [6] where: $\omega_{a}=\omega_{f}-k_{0} y_{c}$.

Sticking can be predicted by calculation of the tangential forces, however these are somewhat uncertain because heat and lubrication from cooling fluids affect the friction force 
and the current flow stress through the material changes based on accumulated plastic strain, temperature and strain rate. Several studies of feed rate (the rate at which the mandrel roll gap is closed) for optimal uniform ring rolling, and, in industrial settings, trial and error can provide a strong set of rules for avoiding sticking. Here, it is proposed that the controller control the ring's rotation rate, and if a sticking condition is encountered, the roll gap is increased to continue forming if the ring speed falls below a threshold angular velocity.

$\omega_{f}$ is the primary actuation for the mean angular velocity of the ring, $\dot{\theta}_{p}$. If the ring begins to stick, the controller needs to avoid excessive slip by ensuring the tangential speed of the forming roller is not significantly faster than the tangential speed of the ring. The control law used for this is shown in Algorithm 2, which controls $\theta_{p}$ with a PI controller unless the slip velocity is too high. In practice, sticking may be

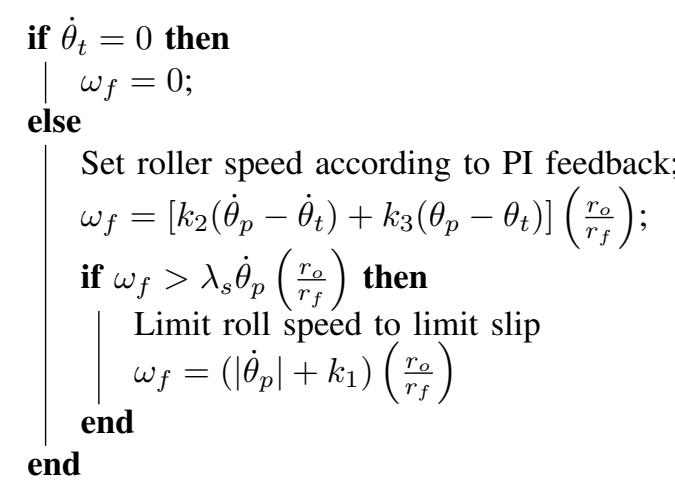

Algorithm 2: Control law for ring rotation angle, $\theta_{p}$. Where $r_{o}$ is the current outer radius of the ring and $r_{f}$ is the forming roll radius. Typically $\lambda_{s}=0.8$.

overcome without opening the roll gap, but often the roll gap has to be increased to proceed. The control law to correct sticking is shown in Algorithm 3, which opens the relevant roll gap slowly until the rotation speed is sufficient to continue. The state of ring geometry is updated constantly from the the camera sensor. The height of a segment is estimated by the axial roll gap during its last pass through the axial rollers and the the predicted strain ratio $\gamma$ if it passed through the radial roll gap too. The model of the compression process described in Section III-B is used to predict the roll gap positions required for the upcoming segments. If the actuators are incapable of moving fast enough, the ring's rotational velocity is reduced to accomodate them. Where segments are obscured in the axial roll gap, the model is required to predict the positions of segments entering that gap.

\section{Final shape recognition}

The ring is rotated and the segments compressed until the target size is achieved within a specified tolerance. The deformation is stopped by setting $w_{f}=0$.

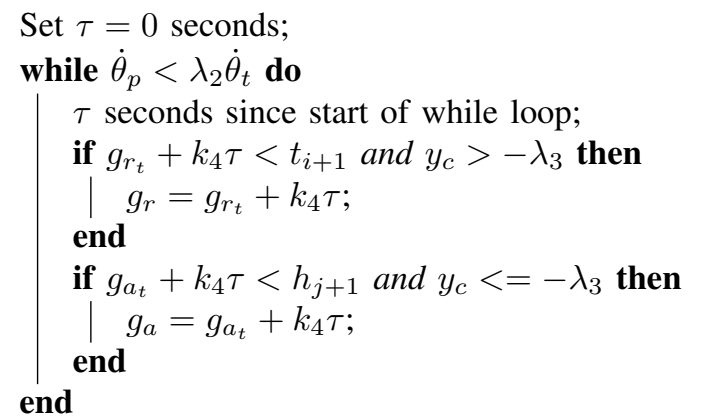

Algorithm 3: Control law to correct for sticking condition. Where $g_{r_{t}}$ and $g_{a_{t}}$ are the current radial and axial roll gap targets. Typically $\lambda_{2}=0.1$. The condition on $y_{c}$ determines which roll gap has locked (if $y_{c}$ is close to zero or positive then the axial rollers are not locked). The if statements check that the roll gaps aren't being opened beyond the size of the next segment (and hence letting go of the ring. $k_{4}$ determines how quickly the roll gaps open.

\section{RESULTS FROM DESKTOP-SCALE EXPERIMENTS}

The sensing strategy in this work has been implemented and tested on a desktop-scale experimental ring rolling machine. Plasticine has been used as a model material for hot-rolled rings. A bench mark target ring shape - with constant axial thickness and a section spanning $\pi / 4$ radians having a larger radial thickness than the rest - was used. Figure 7 demonstrates the initial stages of the forming, and intermediate state and the state near completion.

\section{CONCLUSIONS}

The radial-axial ring rolling forging process can give rise to complex changes in geometry for rolled products during forming. This paper has presented: a novel sensing technique for measuring the changing geometry; a simplifying volumetric model for the storing of geometric data and the prediction of shape changes; and control laws for achieving the targeted product shape in a specified time with fault detection.

The sensing technique permits the tracking of the displacement of the upper surface of a deforming ring in real-time. This broad measurement allows the full state of the outer surface of the ring geometry to be estimated with high resolution and sufficient frequency for the control requirements. These data are essential for the control of variable geometry in a feedback control system. The sensing strategy has been implemented and proved using a desktop-scale ring rolling machine.

The volumetric model is required to calculate the location of the material currently being deformed by the rollers with respect to the larger body of the product. An estimate of the material flow in the ring is essential for finding the actuator inputs necessary to move the shape towards the final target.

The control laws are based on PID control of various degrees of freedom within the ring rolling process. These feedback controls are bounded by strict limits, which are based on preventing loss of control and irreparable damage 

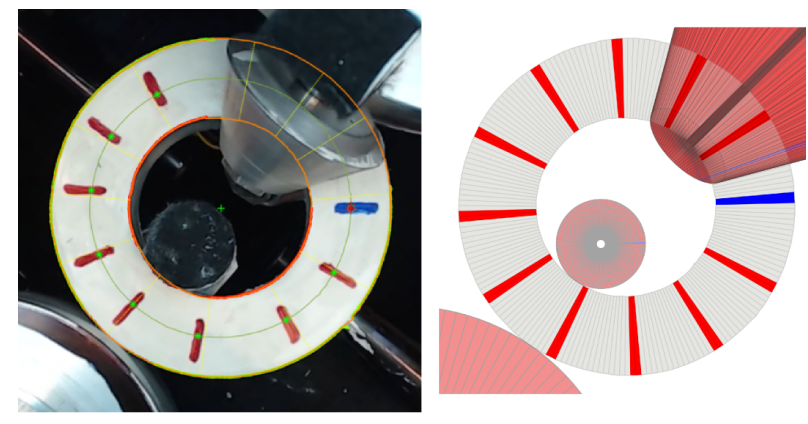

Initial ring state
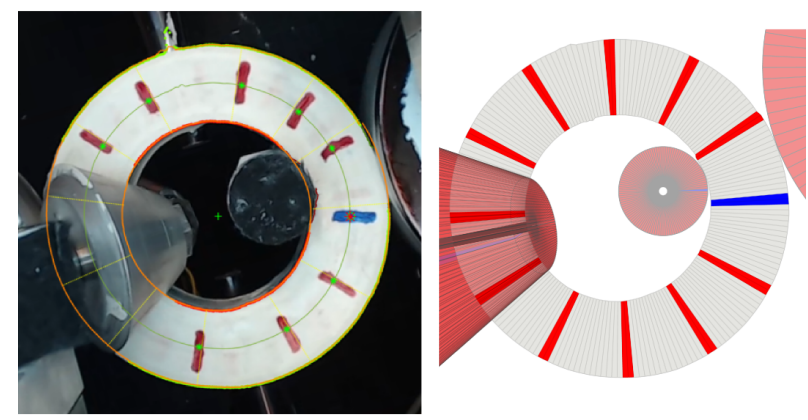

Intermediate ring state
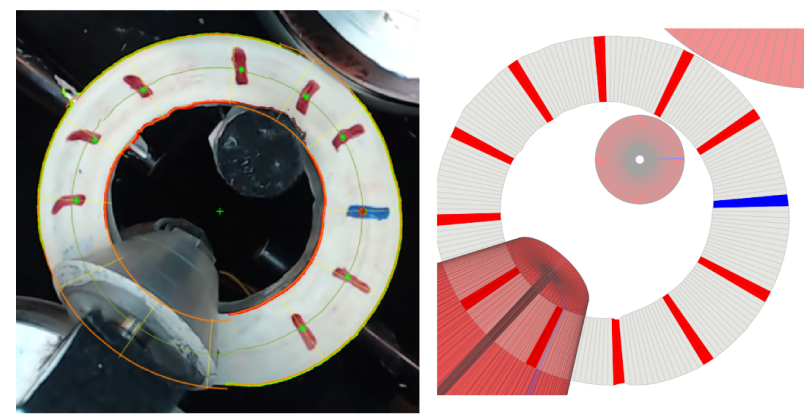

Final ring state

Fig. 7. The plasticine ring undergoing forming with a targeted shape of $\pi / 8$ thicker than the rest of the ring. Initially the ring had internal and external radii of $20 \mathrm{~mm}$ and $37.5 \mathrm{~mm}$ respectively. It's axial height was 20 $\mathrm{mm}$. All diagrams are shown at the same scale.

to the ring product. The control laws are also capable of detecting the sticking fault, where the ring stops rotating through one of the roll gaps due to an over-compression. A controller designed according to this scheme would be capable of forming a ring with variable wall thickness and height, or of correcting a ring with irregular shape into a uniform cross section. Future work will prove these laws are sufficient to generate a variety of target ring shapes.

\section{ACKNOWLEDGMENT}

The authors would like to thank Jianglin Huang for his supporting modelling work and Vahid Jenkouk for his guidance on ring rolling processes.

This work was supported by the Engineering and Physical Sciences Research Council (EPSRC).

\section{REFERENCES}

[1] "Euroforge : 2011 world-wide survey," 2011.

[2] ASM Metals Handbook Volume 14: Forming and Forging. ASM International, 1988

[3] J. M. Allwood, A. E. Tekkaya, and T. F. Stanistreet, "The development of ring rolling technology," Steel research international, vol. 76, no. 2-3, pp. 111-120, 2005.

[4] B. H. Amstead, P. F. Ostwald, and M. L. Begeman, Manufacturing processes. Wiley, 1987.

[5] S. Kalpakjian and S. Schmid, Manufacturing Engineering \& Technology. Pearson Education, 2013.

[6] M. R. Arthington, C. Cleaver, J. Allwood, and S. Duncan, "Real-time measurement of ring-rolling geometry using low-cost hardware," in 2014 UKACC International Conference on Control (CONTROL), no. July. IEEE, July 2014, pp. 603-608.

[7] H. D. CHOI and H. S. CHO, "On the dynamic characteristics of radialaxial ring rolling processes," Journal of engineering for industry, vol. 114, no. 2, pp. 188-195, 1992.

[8] D. Yang and K. Kim, "Rigid-plastic finite element analysis of plane strain ring rolling," International Journal of Mechanical Sciences, vol. 30, no. 8, pp. 571-580, Jan. 1988.

[9] N. Kim, S. Machida, and S. Kobayashi, "Ring rolling process simulation by the three dimensional finite element method," International Journal of Machine Tools and Manufacture, vol. 30, no. 4, pp. 569577, Jan. 1990.

[10] V. Jenkouk, G. Hirt, M. Franzke, and T. Zhang, "Finite element analysis of the ring rolling process with integrated closed-loop control," CIRP Annals - Manufacturing Technology, vol. 61, no. 1, pp. 267-270, Jan. 2012.

[11] H. Choi and H. Cho, "An Adaptive Control Approach to the Ring Geometry Control for Radial-Axial Ring Rolling Processes," in Proceedings of the Institution of Mechanical Engineers, 1989, pp. 243254.

[12] F.-L. Yan, L. Hua, and Y.-Q. Wu, "Planning feed speed in cold ring rolling," International Journal of Machine Tools and Manufacture, vol. 47, no. 11, pp. 1695-1701, Sept. 2007.

[13] H. Lin and R. Wei, "Design and simulation of feed speed controller in ring rolling process," in 9th International Conference on Technology of Plasticity, ICTP 2008. Hanrimwon Publishing Co., 2008, pp. 15251530

[14] K.-H. Lee and B.-M. Kim, "Advanced feasible forming condition for reducing ring spreads in radialaxial ring rolling," International Journal of Mechanical Sciences, vol. 76, pp. 21-32, Nov. 2013.

[15] W. Johnson and G. Needham, "Plastic hinges in ring indentation in relation to ring rolling," International Journal of Mechanical Sciences, vol. 10, no. 6, pp. 487-490, June 1968.

[16] A. Kluge, Y.-H. Lee, H. Wiegels, and R. Kopp, "Control of strain and temperature distribution in the ring rolling process," Journal of Materials Processing Technology, vol. 45, no. 1-4, pp. 137-141, Sept. 1994.

[17] H. K. Moon, M. C. Lee, and M. S. Joun, "Predicting polygonal-shaped defects during hot ring rolling using a rigid-viscoplastic finite element method," International Journal of Mechanical Sciences, vol. 50, no. 2, pp. 306-314, Feb. 2008. 